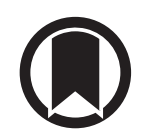

CrossMark

\title{
Pharmacokinetics of tuberculosis drugs in HIV-infected patients from Irkutsk, Russian Federation: redefining drug activity
}

\author{
To the Editor:
}

The Russian Federation has the third highest burden of multidrug-resistant (MDR) tuberculosis (TB) in the world, accounting for $10.5 \%$ of cases worldwide [1]. Management of the drug-resistant TB epidemic in the Siberian province of Irkutsk is further complicated by high rates of HIV co-infection [2], which leads to early mortality and risk for acquired Mycobacterium tuberculosis drug resistance [3]. Such poor treatment outcomes may be in part a consequence of pharmacokinetic variability rendering subtherapeutic drug concentrations [4-6]. Serum area under the concentration curve (AUC) is the pharmacokinetic parameter correlative with efficacy for most concentration-dependent TB drugs $[7,8]$ and AUC/minimum inhibitory concentration (MIC) ratio can be further used to predict treatment response [9]. However, relatively few studies have examined the pharmacokinetics of MDR-TB drugs, and none from HIV-infected patients in the Russian Federation. The following prospective cohort study of HIV-TB co-infected patients in Irkutsk was performed to describe pharmacokinetic variability and MIC ranges, and determine if drug activity associates with treatment response. The setting was hypothesised to be particularly informative given the high incidence of MDR- and extensively drug-resistant (XDR)-TB and routine use of novel treatment strategies, including high-dose isoniazid.

Consecutive HIV-infected patients initiating TB treatment at the Irkutsk Regional TB Referral Hospital were recruited, with an aim of 70 patients to enrol at least 30 patients treated for MDR-TB. All eligible patients provided written informed consent and the protocol was approved by the institutional review boards of the Scientific Centre for Family Health and Human Reproduction Problems in Irkutsk, and of the University of Virginia (Charlottesville, VA, USA). After 2 weeks of treatment, medications were directly administered and plasma samples collected at $2 \mathrm{~h}$ and $6 \mathrm{~h}$ after administration. Pyrazinamide was dosed separately from morning medications and only 2-h concentrations were measured. Samples were immediately centrifuged and the serum stored at $-80^{\circ} \mathrm{C}$ prior to a batched dry-ice shipment to the University of Florida Infectious Disease Pharmacokinetics Laboratory (Gainesville, FL, USA). Drug concentrations were measured using validated liquid chromatography-mass spectrometry assays. Peak concentration (Cmax) was defined as the highest value obtained in the dosing interval, and AUC from 0 to $6 \mathrm{~h}$ (AUC0-6) was calculated using Phoenix WinNonlin software v.7.0 (Certara, Princeton, NJ, USA). Conventional $M$. tuberculosis drug susceptibility results that the clinicians used for initial drug regimens were obtained by chart review. MIC testing was performed for study purposes using the MYCOTB Sensititre plate (Trek Diagnostic Systems, Oakwood Village, OH, USA) [9]. For redefining drug activity, a drug was classified as active when Cmax was greater than individual isolate MIC, or when Cmax was greater than the median MIC for the cohort when the individual isolate MIC was not available. Outcomes were reported as treatment success in the event of a cure or completion of treatment with symptomatic and radiographic improvement. Treatment failure was defined as death or inability to complete treatment.

Data were entered into Microsoft Excel and analysed using SPSS v22 (IBM Corp., Armonk, NY, USA). Comparisons of means and medians were by two-sample t-test and Kruskal-Wallis respectively. Tests of significance were two-tailed. For hypothesis generation of the effects of drug activity with treatment

@ERSpublications

In Irkutsk, drug concentration testing of TB medications can improve outcomes in HIV-TB co-infected patients http://ow.ly/bj4a30jkqum

Cite this article as: Lyles $\mathrm{G}$, Ogarkov $\mathrm{O}$, Zhdanova $\mathrm{S}$, et al. Pharmacokinetics of tuberculosis drugs in HIV-infected patients from Irkutsk, Russian Federation: redefining drug activity. Eur Respir J 2018; 51: 1800109 [https://doi.org/10.1183/13993003.00109-2018]. 
TABLE 1 Pharmacokinetics and susceptibilities for tuberculosis (TB) drugs in HIV-TB co-infected patients from Irkutsk

\begin{tabular}{|c|c|c|c|c|c|c|c|c|c|}
\hline Drug & Patients n & Dose $\mathbf{m g} \cdot \mathbf{k g}^{-1}$ & $\begin{array}{l}\text { Recommended } \\
\text { dose }\end{array}$ & $\mathrm{C}_{\max } \mu \mathrm{g} \cdot \mathrm{mL}^{-1}$ & $\begin{array}{l}\text { Expected } C_{\max } \\
\mu \mathrm{g} \cdot \mathrm{mL}^{-1}\end{array}$ & $A U C_{0-6} \mu \mathrm{g} \cdot \mathrm{mL}^{-1}$ & $\mathrm{MIC}^{\#} \mu \mathrm{g} \cdot \mathrm{mL}^{-1}$ & $\begin{array}{l}\text { Susceptible } \\
\text { by DST }\end{array}$ & $\begin{array}{c}\text { Susceptible } \\
\text { by } C_{\max } \text { or MIC }\end{array}$ \\
\hline Isoniazid & 38 & $9.7(8.9-10.0)$ & $4-6 \mathrm{mg} \cdot \mathrm{kg}^{-1}$ & 3.1 (trace-5.47) & $3-6$ & $15.5(6.2-24.5)$ & $2.0(0.81-4)$ & $14(36.8)$ & $22(57.9)$ \\
\hline Levofloxacin & 30 & 9.3 (8.7-11.2) & $750-1000 \mathrm{mg}$ & $3.7(0.05-6.47)$ & $8-12$ & $16.5(0.2-33.5)$ & $0.5(0.5-1.0)$ & $26(86.7)$ & 19 (63.3) \\
\hline Moxifloxacin & 7 & $9.7(7.8-10.7)$ & $400-800 \mathrm{mg}$ & 0.46 (trace-4.39) & $3-5$ & $11.1(4.0-15.2)$ & $0.25(0.12-0.37)$ & $6(85.7)$ & $4(57.1)$ \\
\hline Ofloxacin & 19 & $7.6(5.7-8.9)^{+}$ & $800-1000 \mathrm{mg}$ & $4.3(2.3-5.6)$ & $8-12$ & $23.0(10.4-28.0)$ & $0.5(0.5-1.0)$ & $13(68.4)$ & 15 (78.9) \\
\hline Amikacin & 8 & $15.0(13.6-17.6)$ & $15 \mathrm{mg} \cdot \mathrm{kg}^{-1}$ & $35.9(18.1-38.5)$ & $35-45$ & $158.8(87.7-183.6)$ & $0.5(0.25-0.63)$ & 7 (87.5) & $5(62.5)$ \\
\hline Kanamycin & 22 & $16.6(14.1-20.0)$ & $15 \mathrm{mg} \cdot \mathrm{kg}^{-1}$ & $32.9(15.1-49.1)$ & $35-45$ & $173.6(93.5-208.7)$ & $1.2(1.2-15.0)$ & 19 (86.4) & $12(54.5)$ \\
\hline Capreomycin & 18 & $16.5(15.7-18.3)$ & $15 \mathrm{mg} \cdot \mathrm{kg}^{-1}$ & $11.1(5.6-15.8)$ & $35-45$ & $47.7(6.5-74.8)$ & NA & 17 (94.4) & 17 (94.4) \\
\hline Pyrazinamide & 54 & $26.9(25.8-31.3)$ & $25 \mathrm{mg} \cdot \mathrm{kg}^{-1}$ & $19.8(6.9-34.9)$ & $20-60$ & NA & NA & 47 (87.0) & $30(55.6)$ \\
\hline
\end{tabular}

Data are presented as median (interquartile range) or $\mathrm{n}(\%)$, unless otherwise stated. Cmax: peak concentration; AUC0-6: area under the concentration curve from 0 to 6 h; MIC: minimum inhibitory concentration; DST: drug susceptibility testing; NA: not available. \#: MIC testing for isoniazid, levofloxacin, ofloxacin and amikacin was available for 34 patients, and for moxifloxacin and kanamycin for 33 patients; capreomycin and pyrazinamide MICs were not performed as they were not part of the version of the MIC plate. ": DST by conventional phenotypic test per hospital protocol and/or chart review. ${ }^{+}$: ofloxacin was administered at this dose twice a day. 
outcome, a decision tree method, classification and regression tree (CART), was applied (RStudio, Inc., Boston, MA, USA). Treatment outcome was the dependent variable while predictors included demographics, the presence of central nervous system (CNS) TB, cavitary disease on chest imaging, treatment with antiretrovirals prior to TB treatment initiation, Cmax and AUC values for individual drugs and the redefined total number of active drugs in a patient's regimen based on the pharmacokinetic data.

A total of 69 patients with HIV had pharmacokinetic testing at 2 weeks after treatment initiation. Mean \pm SD age was $34 \pm 6.2$ years, 45 (65.2\%) were male. Mean \pm SD CD4 count was $180 \pm 202$ cells $\cdot \mathrm{mL}^{-1}$, median HIV viral load was 182686 (interquartile range (IQR) 5200-822067). Only 19 (27.5\%) patients were on antiretrovirals at TB treatment initiation. 36 (52.2\%) patients had prior TB treatment, 51 (73.9\%) were smokers, 31 (44.9\%) used intravenous drugs, 11 (15.9\%) had CNS TB and 25 (36.2\%) had cavitary disease. Of the total, 36 (52.2\%) were classified as having drug-susceptible TB, 10 (14.5\%) MDR-TB, 17 (24.6\%) pre-XDR-TB and six (8.7\%) XDR-TB. MIC testing was performed on 34 patients' M. tuberculosis isolates. All patients were inpatients for the duration of the study procedures.

A total of 38 patients were treated with isoniazid, with a calculated median (IQR) dose of 9.7 (8.910.0) $\mathrm{mg} \cdot \mathrm{kg}^{-1}$ (table 1). Fluoroquinolones were used in 54 cases; of those, levofloxacin and moxifloxacin were used at standard doses while the ofloxacin median dose was $400 \mathrm{mg}$ twice per day (median dose $7.6 \mathrm{mg} \cdot \mathrm{kg}^{-1}$ ) (table 1). Cmax values were frequently below expected ranges, including trace values for the orally administered drugs. Drug activity was commonly redefined as active for those dosed with isoniazid, and more commonly redefined as inactive for those dosed with fluoroquinolones and injectable agents (table 1).

Based on the Cmax and MIC informed drug activity, patients were treated with a significantly lower number of active drugs $(3.25 \pm 1.40)$ compared to the number of drugs presumed to be active when initially prescribed $(4.81 \pm 0.94 ; \mathrm{p}<0.001) .50$ patients had treatment outcomes that could be accurately categorised, with $16(32.0 \%)$ experiencing treatment failure. In decision tree analysis, CNS TB was the most important predictor of treatment failure. However, when CNS TB was removed as a predictor, having $<4.5$ active drugs, as redefined by $\mathrm{Cmax}$ and MIC testing, was the first classification of significance, correctly identifying 15 (93\%) out of the 16 patients with treatment failure.

The treatment approach to drug-resistant TB has targeted shorter durations, better-tolerated regimens and drug susceptibility testing. Our findings from this HIV-infected cohort in Irkutsk with complex M. tuberculosis drug resistance patterns suggest that a more comprehensive definition of drug activity, which incorporates circulatory drug exposure relative to quantitative susceptibility testing of the patient's M. tuberculosis isolate, may further improve treatment outcomes. Potential alterations in drug and dose selection occurre across multiple drug classes.

Isoniazid was commonly prescribed despite conventional $M$. tuberculosis drug resistance, but given at a median dose of $10 \mathrm{mg} \cdot \mathrm{kg}^{-1}$, double that given for isoniazid-susceptible M. tuberculosis [10]. Despite considerable pharmacokinetic variability in estimated Cmax and AUC for higher-dose isoniazid, many patients achieved a serum concentration that exceeded the MIC. The World Health Organization has recently approved a 9-month treatment regimen that utilises higher-dose isoniazid, hypothesised to result in increased drug concentration at the site of TB disease to overcome low-level resistance [11]. In previous studies from Irkutsk we have noted the M. tuberculosis inhA promoter mutation to be rare compared to the $k a t G$ catalase mutation, those mutations conferring low- and high-level isoniazid resistance, respectively [12]. Yet the MIC data from this study suggest a greater variability in quantitative isoniazid resistance, which probably explains why higher-dose isoniazid and improved serum exposure may result in clinical efficacy.

For the fluoroquinolones, human studies have demonstrated a dose-dependent increase in concentrations in M. tuberculosis-infected lung lesions, suggesting that increasing serum concentrations may lead to better treatment outcomes $[13,14]$. The optimal serum exposure relative to the MIC for predicting treatment efficacy has not been adequately studied, but our findings from Irkutsk would suggest that most patients were significantly under-dosed, even for patients with conventionally fluoroquinolone-susceptible M. tuberculosis. Similar patterns to the fluoroquinolones were observed for the injectable agents. However, given that fewer than expected patients were initiated on antiretrovirals at the time of blood collection for pharmacokinetic testing, drug-drug interactions were not investigated.

Nevertheless, our hypothesis-generating decision tree analysis found a break-point of 4.5 active drugs was most predictive of favourable outcome. In practice in Irkutsk and other drug-resistant TB endemic settings, most patients are initiated on treatment with a rapid molecular result for rifampin resistance from sputum. In order to increase the likelihood of prescribing four or more active drugs, our findings suggest an initial drug regimen in Irkutsk should include higher-dose isoniazid, later-generation fluoroquinolones 
and an expanded regimen for XDR-TB, followed by serum concentration testing of medications prone to pharmacokinetic variability. Guidance for such an approach exists [15], and we recommend implementation projects to test the recommendations.

Galina Lyles ${ }^{1}$, Oleg Ogarkov ${ }^{2,3,4}$, Svetlana Zhdanova $^{2}$, Charles A. Peloquin ${ }^{5}$, Andrew Ebers ${ }^{6}$, Herman Pfaeffle Mohammad H. Al-Shaer $\odot^{5}$, Elena Moiseeva ${ }^{4}$, Elena Zorkaltseva ${ }^{3,4}$, Mikhail Koscheev ${ }^{4}$, Eric R. Houpt ${ }^{6}$ and Scott K. Heysell ${ }^{6}$

${ }^{1}$ Division of Pulmonary and Critical Care Medicine, University of Virginia, Charlottesville, VA, USA. ${ }^{2}$ Scientific Centre for Family Health and Human Reproduction Problems, Irkutsk, Russia. ${ }^{3}$ Irkutsk State Medical Academy of Continuing Education, Irkutsk, Russia. ${ }^{4}$ Irkutsk Clinical Tuberculosis Hospital, Irkutsk, Russia. ${ }^{5}$ College of Pharmacy, University of Florida, Gainesville, FL, USA. 'Division of Infectious Diseases and International Health, University of Virginia, Charlottesville, VA, USA. ${ }^{7}$ School of Medicine, University of Virginia, Charlottesville, VA, USA.

Correspondence: Galina Lyles, Division of Pulmonary and Critical Care Medicine, University of Virginia, P.O. Box 800546, Charlottesville, VA 22908, USA. E-mail: galina.lyles@gmail.com

Received: Jan 172018 | Accepted after revision: March 132018

Conflict of interest: None declared.

Support statement: This work was supported by National Institutes of Health grants R21 AI108521 and U01 AI119954, and Russian Foundation for Basic Research grant 17-54-30020. Funding information for this article has been deposited with the Crossref Funder Registry.

\section{References}

1 World Health Organization. Global Tuberculosis Report 2017. WHO/HTM/TB/2017.23. Geneva, World Health Organization, 2017. Available from: www.who.int/tb/publications/global_report/en/

2 Heysell SK, Ogarkov OB, Zhdanova S, et al. Undertreated HIV and drug-resistant tuberculosis at a referral hospital in Irkutsk, Siberia. Int J Tuberc Lung Dis 2016; 20: 187-192.

3 Gandhi NR, Shah NS, Andrews JR, et al. HIV coinfection in multidrug- and extensively drug-resistant tuberculosis results in high early mortality. Am J Respir Crit Care Med 2010; 181: 80-86.

4 Weiner M, Burman W, Vernon A, et al. Low isoniazid concentrations and outcome of tuberculosis treatment with once-weekly isoniazid and rifapentine. Am J Respir Crit Care Med 2003; 167: 1341-1347.

5 Vinnard C, Ravimohan S, Tamuhla N, et al. Isoniazid clearance is impaired among human immunodeficiency virus/tuberculosis patients with high levels of immune activation. Br J Clin Pharmacol 2017; 83: 801-811.

6 Alsultan A, Peloquin CA. Therapeutic drug monitoring in the treatment of tuberculosis: an update. Drugs 2014; 74: 839-854.

7 Pasipanodya JG, McIlleron H, Burger A, et al. Serum drug concentrations predictive of pulmonary tuberculosis outcomes. J Infect Dis 2013; 208: 1464-1473.

8 Alffenaar JC, Akkerman OW, Anthony RM, et al. Individualizing management of extensively drug-resistant tuberculosis: diagnostics, treatment, and biomarkers. Expert Rev Anti Infect Ther 2017; 15: 11-21.

9 Heysell SK, Ahmed S, Ferdous SS, et al. Quantitative drug-susceptibility in patients treated for multidrug-resistant tuberculosis in Bangladesh: implications for regimen choice. PLoS One 2015; 10: e0116795.

10 Falzon D, Schünemann HJ, Harausz E, et al. World Health Organization treatment guidelines for drug-resistant tuberculosis, 2016 update. Eur Respir J 2017; 49: 1602308.

11 Van Deun A, Maug AK, Salim MA, et al. Short, highly effective, and inexpensive standardized treatment of multidrug-resistant tuberculosis. Am J Respir Crit Care Med 2010; 182: 684-692.

12 Zhdanova S, Heysell SK, Ogarkov O, et al. Primary multidrug-resistant Mycobacterium tuberculosis in 2 regions, Eastern Siberia, Russian Federation. Emerg Infect Dis 2013; 19: 1649-1652.

13 Kempker RR, Barth $\mathrm{AB}$, Vashakidze S, et al. Cavitary penetration of levofloxacin among patients with multidrug-resistant tuberculosis. Antimicrob Agents Chemother 2015; 59: 3149-3155.

14 Davies Forsman L, Bruchfeld J, Alffenaar JC. Therapeutic drug monitoring to prevent acquired drug resistance of fluoroquinolones in the treatment of tuberculosis. Eur Respir J 2017; 49: 1700173.

15 Ghimire S, Bolhuis MS, Sturkenboom MG, et al. Incorporating therapeutic drug monitoring into the World Health Organization hierarchy of tuberculosis diagnostics. Eur Respir J 2016; 47: 1867-1869. 